\title{
Article
}

\section{On Some NeutroHyperstructures}

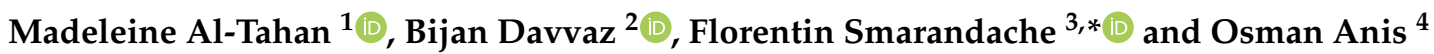 \\ 1 Department of Mathematics, Lebanese International University, Bekaa 1803, Lebanon; \\ madeline.tahan@liu.edu.lb \\ 2 Department of Mathematics, Yazd University, Yazd 89139, Iran; davvaz@yazd.ac.ir \\ 3 Mathematics and Science Division, Gallup Campus, University of New Mexico, 705 Gurley Ave., \\ Gallup, NM 87301, USA \\ 4 Department of Mathematics, Lebanese International University, Akkar 35111, Lebanon; \\ osman.anis@liu.edu.lb \\ * Correspondence: smarand@unm.edu
}

Citation: Al-Tahan, M.; Davvaz, B.; Smarandache, F.; Anis, O. On Some NeutroHyperstructures. Symmetry 2021, 13, 535. https://doi.org/ 10.3390/sym13040535

Academic Editor: Palle E. T. Jorgensen

Received: 20 February 2021

Accepted: 23 March 2021

Published: 25 March 2021

Publisher's Note: MDPI stays neutral with regard to jurisdictional claims in published maps and institutional affiliations.

Copyright: (c) 2021 by the authors. Licensee MDPI, Basel, Switzerland. This article is an open access article distributed under the terms and conditions of the Creative Commons Attribution (CC BY) license (https:// creativecommons.org/licenses/by/ $4.0 /)$.

\begin{abstract}
Neutrosophy, the study of neutralities, is a new branch of Philosophy that has applications in many different fields of science. Inspired by the idea of Neutrosophy, Smarandache introduced NeutroAlgebraicStructures (or NeutroAlgebras) by allowing the partiality and indeterminacy to be included in the structures' operations and/or axioms. The aim of this paper is to combine the concept of Neutrosophy with hyperstructures theory. In this regard, we introduce NeutroSemihypergroups as well as $\mathrm{NeutroH}_{v}$-Semigroups and study their properties by providing several illustrative examples.
\end{abstract}

Keywords: NeutroHypergroupoid; NeutroSemihypergroup; $\mathrm{NeutroH}_{v}$-semigroup; NeutroHyperideal; NeutroStrongIsomorphism

MSC: 03A99; 03G99; 20N20

\section{Introduction}

In 1995 and inspired by the existence of neutralities, Smarandache introduced Neutrosophy as a new branch of Philosophy that deals with indeterminacy. During the past, ideas were viewed as "True" or "False"; however, if we view an idea from a neutrosophic point of view, it will be "True", "False", or "Indeterminate". The indeterminacy is the key that distinguishes Neutrosophy from other approaches. In the past twenty years, this field demonstrated important progress in which it grabbed the attention of many researchers and different works were done from both a theoretical point of view and from an applicative view. Unlike our real world that is full of imperfections and partialities, abstract systems are constructed on a given perfect space (set), where the operations are totally well-defined and the axioms are totally true for all spacial elements. Starting from the latter idea, Smarandache [1-3] introduced NeutroAlgebra, whose operations are partially well-defined, partially indeterminate, and partially outer-defined, and the axioms are partially true, partially indeterminate, and partially false. Many researchers worked on special types of NeutroAlgebras by applying them to different types of algebraic structures such as groups, rings, $B E$-Algebras, $B C K$-Algebras, etc. For more details, we refer to [4-10].

On the other hand, hyperstructure theory is a generalization of classical algebraic structures and was introduced in 1934 at the eighth Congress of Scandinavian Mathematicians by Marty [11]. Marty generalized the notion of groups by defining hypergroups. The class of algebraic hyperstructures is larger than that of algebraic structures where the operation on two elements in the latter is again an element, whereas the hyperoperation of two elements in the first class is a non-void set. For details about hyperstructure theory and its applications, we refer to the articles [12-15] and the books [16-18]. A generalization of algebraic hyperstructures, known as weak hyperstructures ( $H_{v}$-structures), was introduced 
in 1994 by Vougiouklis [19]. The axioms in the latter are weaker than that of algebraic hyperstructures. For details about $H_{v}$-structures, we refer to [19-22].

As a natural extension of NeutroAlgebraicStructure, NeutroHyperstructure was defined recently [23,24] where Ibrahim and Agboola [23] defined NeutroHypergroups and studied a special type. Our paper is concerned about some NeutroHyperstructures and is organized as follows: Section 2 presents some basic preliminaries related to hyperstructure theory. Section 3 defines NeutroSemihypergroups, $\mathrm{NeutroH}_{v}$-Semigroups, and some related new concepts and illustrates these new concepts via examples. Moreover, we study some properties of their subsets under NeutroStrongHomomorphism.

\section{Algebraic Hyperstructures}

In this section, we present some definitions and examples about (weak) algebraic hyperstructures that are used throughout the paper. For more details about hyperstructure theory, we refer to [16-20].

Definition 1 ([16]). Let $H$ be a non-empty set and $\mathcal{P}^{*}(H)$ be the family of all non-empty subsets of $H$. Then, a mapping $\circ: H \times H \rightarrow \mathcal{P}^{*}(H)$ is called a binary hyperoperation on $H$. The couple $(H, O)$ is called a hypergroupoid.

If $A$ and $B$ are two non-empty subsets of $H$ and $h \in H$, then we define:

$$
A \circ B=\bigcup_{\substack{a \in A \\ b \in B}} a \circ b, h \circ A=\{h\} \circ A \text { and } A \circ h=A \circ\{h\} .
$$

A hypergroupoid $(H, \circ)$ is called a semihypergroup if the associative axiom is satisfied. i.e., for every $x, y, z \in H, x \circ(y \circ z)=(x \circ y) \circ z$. In other words,

$$
\bigcup_{u \in y \circ z} x \circ u=\bigcup_{v \in x \circ y} v \circ z .
$$

An element $h$ in a hypergroupoid $(H, \circ)$ is called idempotent if $h \circ h=h$.

Example 1. Let $H$ be any non-empty set and define " $\star$ " on $H$ as follows. For all $x, y \in H$, $x \star y=\{x, y\}$. Then $(H, \star)$ is a semihypergroup.

Example 2. Let $H_{0}=\{e, b, c\}$ and $\left(H_{0},+\right)$ be defined by the following table.

\begin{tabular}{|c|ccc|}
\hline+ & $e$ & $b$ & $c$ \\
\hline$e$ & $e$ & $\{e, b\}$ & $\{e, c\}$ \\
$b$ & $e$ & $\{e, b\}$ & $\{e, c\}$ \\
$c$ & $e$ & $\{e, b\}$ & $\{e, c\}$ \\
\hline
\end{tabular}

Then $\left(H_{0},+\right)$ is a semihypergroup and e is an idempotent element in $H_{0}$.

As a generalization of algebraic hyperstructures, Vougiouklis $[19,20]$ introduced $H_{v^{-}}$ structures. Weak axioms in $H_{v}$-structures replace some axioms of classical algebraic hyperstructures.

Definition $2([19,20])$. A hypergroupoid $(H, O)$ is called an $H_{v}$-semigroup if the weak associative axiom is satisfied. i.e., $(x \circ(y \circ z)) \cap((x \circ y) \circ z) \neq \varnothing$ for all $x, y, z \in H$.

Example 3. Let $H_{1}=\{0,1,2,3\}$ and " + " be the hyperoperation on $H_{1}$ defined by the following table.

\begin{tabular}{|c|cccc|}
\hline+ & 0 & 1 & 2 & 3 \\
\hline 0 & 0 & 1 & $\{0,2\}$ & 3 \\
1 & 1 & 2 & 3 & 0 \\
2 & 2 & 3 & 0 & 1 \\
3 & 3 & 0 & 1 & 2 \\
\hline
\end{tabular}


Then $\left(H_{1},+\right)$ is an $H_{v}$-semigroup.

Remark 1. Every semigroup is a semihypergroup and every semihypergroup is an $H_{v}$-semigroup.

Definition 3 ([17]). Let $(H, \circ)$ be a semihypergroup $\left(H_{v}\right.$-semigroup $)$ and $M \neq \varnothing \subseteq H$. Then $M$ is $a$

1. subsemihypergroup ( $H_{v}$-subsemigroup) of $H$ if $(M, 0)$ is a semihypergroup $\left(H_{v}\right.$-semigroup).

2. left hyperideal of $H$ if $M$ is a subsemihypergroup $\left(H_{v}\right.$-subsemigroup) of $H$ and $h \circ a \subseteq M$ for all $h \in H$.

3. right hyperideal of $H$ if $M$ is a subsemihypergroup $\left(H_{v}\right.$-subsemigroup) of $H$ and $a \circ h \subseteq M$ for all $h \in H$.

4. hyperideal of $H$ if $M$ is both: a left hyperideal of $H$ and a right hyperideal of $H$.

Remark 2. Let $(H, \circ)$ be a semihypergroup $\left(H_{v}\right.$-semigroup) and $M \neq \varnothing \subseteq H$. To prove that $M$ is subsemihypergroup ( $H_{v}$-subsemigroup) of $H$, it suffices to show that $a \circ b \subseteq M$ for all $a, b \in M$.

\section{NeutroHyperstructures}

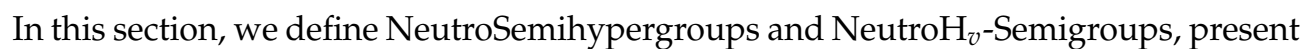
some illustrative examples, and study several properties of some important subsets of NeutroSemihypergroups and $\mathrm{NeutroH}_{v}$-Semigroups.

Definition 4. Let $A$ be any non-empty set and "." be a hyperoperation on $A$. Then "." is called a NeutroHyperoperation on $A$ if some (or all) of the following conditions hold in a way that $(T, I, F) \notin\{(1,0,0),(0,0,1)\}$.

1. There exist $x, y \in A$ with $x \cdot y \subseteq A$. (This condition is called degree of truth, " $T$ ").

2. There exist $x, y \in A$ with $x \cdot y \nsubseteq A$. (This condition is called degree of falsity, " $F ")$.

3. There exist $x, y \in A$ with $x \cdot y$ is indeterminate in $A$. (This condition is called degree of indeterminacy, " $I ")$.

Definition 5. Let $A$ be any non-empty set and "." be a hyperoperation on $A$. Then "." is called an AntiHyperoperation on $A$ if $x \cdot y \nsubseteq A$ for all $x, y \in A$.

Definition 6. Let $A$ be any non-empty set and "." be a hyperoperation on $A$. Then "." is called NeutroAssociative on $A$ if there exist $x, y, z, a, b, c, e, f, g \in A$ satisfying some (or all) of the following conditions in a way that $(T, I, F) \notin\{(1,0,0),(0,0,1)\}$.

1. $x \cdot(y \cdot z)=(x \cdot y) \cdot z$; (This condition is called degree of truth, " $T$ ").

2. $a \cdot(b \cdot c) \neq(a \cdot b) \cdot c$; (This condition is called degree of falsity, " $F$ ").

3. $e \cdot(f \cdot g)$ is indeterminate or $(e \cdot f) \cdot g$ is indeterminate or we cannot find if $e \cdot(f \cdot g)$ and $(e \cdot f) \cdot g$ are equal. (This condition is called degree of indeterminacy, " $I$ ").

Definition 7. Let $A$ be any non-empty set and "." be a hyperoperation on $A$. Then "." is called AntiAssociative on $A$ if $a \cdot(b \cdot c) \neq(a \cdot b) \cdot c$ for all $a, b, c \in A$.

Definition 8. Let $A$ be any non-empty set and "." be a hyperoperation on A. Then "." is called a NeutroWeakAssociative on $A$ if there exist $x, y, z, a, b, c, e, f, g \in A$ satisfying some (or all) of the following conditions in a way that $(T, I, F) \notin\{(1,0,0),(0,0,1)\}$.

1. $[x \cdot(y \cdot z)] \cap[(x \cdot y) \cdot z] \neq \varnothing$; (This condition is called degree of truth, " $T$ ").

2. $[a \cdot(b \cdot c)] \cap[(a \cdot b) \cdot c]=\varnothing$; (This condition is called degree of falsity, " $F ")$.

3. $e \cdot(f \cdot g)$ is indeterminate or $(e \cdot f) \cdot g$ is indeterminate or we cannot find if $e \cdot(f \cdot g)$ and $(e \cdot f) \cdot g$ have common elements. (This condition is called degree of indeterminacy, " $I$ ").

Definition 9. Let $A$ be a non-empty set and "." be a hyperoperation on $A$. Then $(A, \cdot)$ is called a

1. NeutroHypergroupoid if "." is a NeutroHyperoperation.

2. NeutroSemihypergroup if "." is NeutroAssociative but not an AntiHyperoperation. 


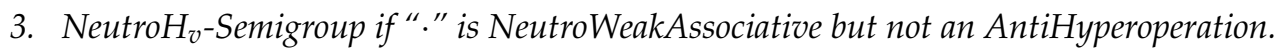

Example 4. Let $A=\{0,1\}$ and $(A,+)$ be defined by the following table.

\begin{tabular}{|c|cc|}
\hline+ & 0 & 1 \\
\hline 0 & $\{0,1\}$ & 0 \\
1 & 1 & 0 \\
\hline
\end{tabular}

Then $(A,+)$ is a NeutroSemihypergroup and $\mathrm{NeutroH}_{v}$-Semigroup. This is clear as

$$
0+(0+0)=\{0,1\}=(0+0)+0 \text { and }(1+1)+1=0 \neq 1=1+(1+1) .
$$

Example 5. Let $\mathbb{R}$ be the set of real numbers and define " $\star$ " on $\mathbb{R}$ as follows.

$$
x \star y= \begin{cases}{[x, y]} & \text { if } x<y ; \\ {[y, x]} & \text { if } y<x ; \\ 0 & \text { if } x=y=0 ; \\ \frac{1}{x} & \text { if } x=y \neq 0 .\end{cases}
$$

Then $(\mathbb{R}, \star)$ is a NeutroSemihypergroup. This is clear as $(1 \star 1) \star 1=1=1 \star(1 \star 1)$ and $(1 \star 2) \star 2=\left\{\frac{1}{2}\right\} \cup[1,2] \neq\left[\frac{1}{2}, 1\right]=1 \star(2 \star 2)$.

Example 6. Let $M=\{m, a, d\}$ and $(M, \cdot)$ be defined by the following table.

\begin{tabular}{|c|ccc|}
\hline$\cdot$ & $m$ & $a$ & $d$ \\
\hline$m$ & $m$ & $m$ & $m$ \\
$a$ & $m$ & $\{m, a\}$ & $d$ \\
$d$ & $m$ & $d$ & $d$ \\
\hline
\end{tabular}

Then $(M, \cdot)$ is a NeutroSemihypergroup. This is clear as $m \cdot(m \cdot m)=m=(m \cdot m) \cdot m$ and $a \cdot(a \cdot d)=d \neq\{m, d\}=(a \cdot a) \cdot d$.

Remark 3. It is well known in classical algebraic hyperstructures that every semihypergroup is a hypergroupoid. This may fail to occur in NeutroHyperstructures. In Example $6,(M, \cdot)$ is a NeutroSemihypergroup that is not a NeutroHypergroupoid.

Proposition 1. Every $H_{v}$-semigroup that is not a semihypergroup and has an idempotent element is a NeutroSemihypergroup.

Proof. Let $(H, \circ)$ be an $H_{v}$-semigroup with $h^{2}=h$ for some $h \in H$. Then $h \circ(h \circ h)=h=$ $(h \circ h) \circ h$. Since $(H, \circ)$ is not a semihypergroup, it follows that there exist $x, y, z \in H$ with $x \circ(y \circ z) \neq(x \circ y) \circ z$. Therefore, $(H, \circ)$ is a NeutroSemihypergroup.

Example 7. Let $M=\{m, a, d\}$ and $(M, \diamond)$ be defined by the following table.

\begin{tabular}{|c|ccc|}
\hline$\diamond$ & $m$ & $a$ & $d$ \\
\hline$m$ & $m$ & $\{a, d\}$ & $d$ \\
$a$ & $\{a, d\}$ & $d$ & $m$ \\
$d$ & $d$ & $m$ & $a$ \\
\hline
\end{tabular}

Then $(M, \diamond)$ is an $H_{v}$-semigroup having $m$ as an idempotent element and hence, it is a NeutroSemihypergroup. 
Remark 4. It is well known in algebraic hyperstructures that every semihypergroup is an $\mathrm{H}_{v^{-}}$ semigroup. This may not hold in NeutroHyperstructures. i.e., A NeutroSemihypergroup may not be a NeutroH $\mathrm{H}_{v}$-Semigroup.

The $H_{v^{-}}$semigroup $(M, \diamond)$ in Example 7 is a NeutroSemihypergroup that is not $\mathrm{NeutroH}_{v^{-}}$ Semigroup.

Example 8. Let $\mathbb{Z}$ be the set of integers and define " $\oplus$ " on $\mathbb{Z}^{2}$ as follows. For all $m, n, p, q \in \mathbb{Z}$,

$$
\begin{aligned}
&(m, 0) \oplus(0,0)=(0,0) \oplus(m, 0)=\{(0,0),(m, 0)\}, \\
&(0, n) \oplus(0,0)=(0,0) \oplus(0, n)=\{(0,0),(0, n)\}, \\
& \text { and if }(n, p, q) \neq(0,0,0),(m, p, q) \neq(0,0,0) \\
&(m, n) \oplus(p, q)=(p, q) \oplus(m, n)=(m+p, n+q) .
\end{aligned}
$$

Then $\left(\mathbb{Z}^{2}, \oplus\right)$ is a NeutroSemihypergroup. This is clear as

$$
[(1,2) \oplus(1,3)] \oplus(1,4)=(3,9)=(1,2) \oplus[(1,3) \oplus(1,4)]
$$

and

$$
[(1,0) \oplus(1,0)] \oplus(0,0)=\{(2,0),(0,0)\} \neq\{(2,0),(1,0),(0,0)\}=(1,0) \oplus[(1,0) \oplus(0,0)] .
$$

Example 9. Let $\mathbb{Z}$ be the set of integers and define " $\odot$ " on $\mathbb{Z}^{2}$ as follows. For all $m, n, p, q \in \mathbb{Z}$,

$$
(m, n) \odot(p, q)= \begin{cases}(m p, n q) & \text { if }(m, n) \neq(1,1) \text { and }(p, q) \neq(1,1) ; \\ \{(p, q),(1,1)\} & \text { if }(m, n)=(1,1) \\ \{(m, n),(1,1)\} & \text { if }(p, q)=(1,1) .\end{cases}
$$

Then $\left(\mathbb{Z}^{2}, \odot\right)$ is a NeutroSemihypergroup. This is clear as

$$
[(1,2) \odot(1,3)] \odot(1,4)=(1,24)=(1,2) \odot[(1,3) \odot(1,4)]
$$

and

$(1,1) \odot[(2,2) \odot(3,3)]=\{(1,1),(6,6)\} \neq\{(1,1),(3,3),(6,6)\}=[(1,1) \odot(2,2)] \odot(3,3)$.

Example 10. Let $\mathbb{Z}_{6}$ be the set of integers under addition modulo 6 and define " $\boxplus$ " on $\mathbb{Z}_{6}$ as follows.

$$
\begin{gathered}
x \boxplus y=(x+y) \quad \bmod 6 \text { for all }(x, y) \notin\{(\overline{0}, \overline{3}),(\overline{0}, \overline{5})\} ， \\
\overline{0} \boxplus \overline{3}=\{\overline{0}, \overline{3}\}, \text { and } \overline{0} \boxplus \overline{5}=\{\overline{0}, \overline{5}\} .
\end{gathered}
$$

Then $\left(\mathbb{Z}_{6}, \boxplus\right)$ is a NeutroSemihypergroup. This is clear as $\overline{0} \boxplus(\overline{0} \boxplus \overline{0})=\overline{0}=(\overline{0} \boxplus \overline{0}) \boxplus \overline{0}$ and $\overline{0} \boxplus(\overline{1} \boxplus \overline{2})=\{\overline{0}, \overline{3}\} \neq \overline{3}=(\overline{0} \boxplus \overline{1}) \boxplus \overline{2}$.

Example 11. Let $M=\{m, a, d\}$ and $(M, \bullet)$ be defined by the following table.

\begin{tabular}{|c|ccc|}
\hline$\bullet$ & $m$ & $a$ & $d$ \\
\hline$m$ & $a$ & $a$ & $d$ \\
$a$ & $\{m, a\}$ & $m$ & $d$ \\
$d$ & $d$ & $d$ & $m$ \\
\hline
\end{tabular}

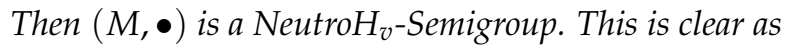

$$
[m \bullet(m \bullet m)] \cap[(m \bullet m) \bullet m]=\{a\} \cap\{m, a\} \neq \varnothing
$$


and

$$
[m \bullet(d \bullet d)] \cap[(m \bullet d) \bullet d]=\{a\} \cap\{m\}=\varnothing .
$$

Moreover, $(M, \bullet)$ is a NeutroSemihypergroup as $d \bullet(d \bullet d)=(d \bullet d) \bullet d$.

Remark 5. Every NeutroSemigroup is both: a NeutroSemihypergroup and a $\mathrm{NeutroH}_{v}$-Semigroup. $\mathrm{So}$, the results related to NeutroSemihypergroups ( $\mathrm{NeutroH}_{v}$-Semigroups) are more general than that related to NeutroSemigroups and as a result, we can deal with NeutroSemigroups as a special case of NeutroSemihypergroups ( $\mathrm{NeutroH}_{v}$-Semigroups).

Example 12. Let $S_{1}=\{s, a, m\}$ and $\left(S_{1}, \cdot 1\right)$ be defined by the following table.

\begin{tabular}{|c|ccc|}
\hline$\cdot 1$ & $s$ & $a$ & $m$ \\
\hline$s$ & $s$ & $m$ & $s$ \\
$a$ & $m$ & $a$ & $m$ \\
$m$ & $m$ & $m$ & $m$ \\
\hline
\end{tabular}

In [6], Al-Tahan et al. proved that $\left(S_{1}, \cdot 1\right)$ is a NeutroSemigroup. Thus, $\left(S_{1}, \cdot 1\right)$ is a NeutroSemihypergroup.

Theorem 1. Let $(H, \circ)$ be a NeutroSemihypergroup (NeutroH $H_{v}$-Semigroup) and " $\star$ " be defined on $H$ as $x \star y=y \circ x$ for all $x, y \in H$. Then $(H, \star)$ is a NeutroSemihypergroup (NeutroH $H_{\mathcal{V}^{-}}$ Semigroup).

Proof. The proof is straightforward.

Example 13. Let $M=\{m, a, d\}$ and $(M, \bullet)$ be the NeutroSemihypergroup defined in Example 11. By applying Theorem 1 , we get that $(M, \circledast)$ defined in the following table is a NeutroSemihypergroup and a $\mathrm{NeutroH}_{v}$-Semigroup.

\begin{tabular}{|c|ccc|}
\hline$\circledast$ & $m$ & $a$ & $d$ \\
\hline$m$ & $a$ & $\{m, a\}$ & $d$ \\
$a$ & $a$ & $m$ & $d$ \\
$d$ & $d$ & $d$ & $m$ \\
\hline
\end{tabular}

Definition 10. Let $(H, \circ)$ be a NeutroSemihypergroup (NeutroH $H_{v}$-Semigroup) and $S \neq \varnothing \subseteq H$. Then $S$ is a NeutroSubsemihypergroup (NeutroH ${ }_{v}$-Subsemigroup) of $H$ if $(S, O)$ is a NeutroSemihypergroup ( $\mathrm{NeutroH}_{v}$-Semigroup).

Remark 6. Let $(H, \circ)$ be a NeutroSemihypergroup (NeutroH $H_{v}$-Semigroup) and $S \neq \varnothing \subseteq H$. Unlike the case in algebraic hyperstructures (Remark 2), proving that $a \circ b \subseteq S$ for all $a, b \in S$ does not imply that $S$ is a NeutroSubsemihypergroup ( $\mathrm{NeutroH}_{v}$-Subsemigroup) of $\mathrm{H}$.

As an illustration of Remark 6, $0 \star 0=\{0\} \subseteq\{0\}$ in Example 5 but $\{0\}$ is not a NeutroSubsemihypergroup of $\mathbb{R}$.

Definition 11. Let $(H, \circ)$ be a NeutroSemihypergroup (NeutroH ${ }_{v}$-Semigroup) and $S \neq \varnothing \subseteq H$ be a NeutroSubsemihypergroup (NeutroH ${ }_{v}$-Subsemigroup). Then

(1) $S$ is a NeutroLeftHyperideal of $H$ if there exists $x \in S$ such that $r \circ x \subseteq S$ for all $r \in H$.

(2) $S$ is a NeutroRightHyperideal of $S$ if there exists $x \in S$ such that $x \circ r \subseteq S$ for all $r \in H$.

(3) $S$ is a NeutroHyperideal of $H$ if there exists $x \in S$ such that $r \circ x \subseteq S$ and $x \circ r \subseteq S$ for all $r \in H$.

A NeutroSemihypergroup ( $\mathrm{NeutroH}_{v}$-Semigroup) is called simple if it has no proper NeutroSubsemihypergroups ( $\mathrm{NeutroH}_{v}$-Subsemigroups). 
Example 14. Let $(A,+)$ be the NeutroSemihypergroup defined in Example 4. Then $A$ is simple. This is clear as $\{0\}$ and $\{1\}$ are the only options for any possible proper NeutroSubsemihypergroup and $(\{0\},+)$ and $(\{1\},+)$ are AntiHypergroupoids.

Example 15. Let $(M, \bullet)$ be the NeutroSemihypergroup defined in Example 11. Then $\{m, a\}$ is a NeutroSubsemihypergroup of $M$.

Example 16. Let $\left(\mathbb{Z}^{2}, \oplus\right)$ be the NeutroSemihypergroup defined in Example $8, M_{1}=\{(x, 0)$ : $x \in \mathbb{Z}\}$, and $M_{2}=\{(0, x): x \in \mathbb{Z}\}$. Then $M_{1}, M_{2}$ are NeutroSubsemihypergroups of $\mathbb{Z}^{2}$.

Remark 7. The intersection of NeutroSubsemihypergroups may fail to be a NeutroSubsemihypergroup. This is clear from Example 16 as $\{(0,0)\}=M_{1} \cap M_{2}$ is not a NeutroSubsemihypergroup of $\mathbb{Z}^{2}$.

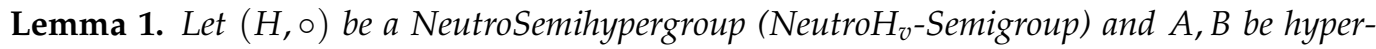
groupoids. If $A, B$ are NeutroSubsemihypergroups ( $\mathrm{NeutroH}_{v}$-Subsemigroups) of $H$ then $A \cup B$ is a NeutroSubsemihypergroup (NeutroH $\mathrm{N}_{v}$-Subsemigroup) of $\mathrm{H}$.

Proof. Let $A, B$ be NeutroSubsemihypergroups. Since $A$ and $B$ are hypergroupoids, it follows that " $\circ$ " is NeutroAssociative on both of $A$ and $B$. The latter implies that there exist $x, y, z, a, b, c, e, f, g \in A \subseteq A \cup B$ satisfying some (or all) of the following conditions in a way that $(T, I, F) \notin\{(1,0,0),(0,0,1)\}$.

1. $T: x \circ(y \circ z)=(x \circ y) \circ z$;

2. $F: a \circ(b \circ c) \neq(a \circ b) \circ c$;

3. $I: e \circ(f \circ g)$ is indeterminate or $(e \circ f) \circ g$ is indeterminate or we cannot find if $e \circ(f \circ g)$ and $(e \circ f) \circ g$ are equal.

Therefore, $A \cup B$ is a NeutroSubsemihypergroup of $H$. The proof of ( $\mathrm{NeutroH}_{v^{-}}$ Subsemigroup is done similarly.

Example 17. Let $\left(\mathbb{Z}^{2}, \odot\right)$ be the NeutroSemihypergroup defined in Example $9, N_{1}=\{(x, y) \in$ $\left.\mathbb{Z}^{2}: x, y \geq 1\right\} \cup\{(0,0)\}$, and $N_{2}=\left\{(x, y) \in \mathbb{Z}^{2}: x, y \leq 1\right\} \cup\{(0,0)\}$. Then $N_{1}, N_{2}$ are NeutroHyperideals of $\mathbb{Z}^{2}$. We show that $N_{1}$ is a NeutroHyperideal of $\mathbb{Z}^{2}$ and $N_{2}$ may be done similarly. Since

$$
[(1,2) \odot(1,3)] \odot(1,4)=(1,24)=(1,2) \odot[(1,3) \odot(1,4)]
$$

and

$$
(1,1) \odot[(2,2) \odot(3,3)]=\{(1,1),(6,6)\} \neq\{(1,1),(3,3),(6,6)\}=[(1,1) \odot(2,2)] \odot(3,3),
$$

it follows that $N_{1}$ is a NeutroSubsemihypergroup of $\mathbb{Z}^{2}$. Having $(0,0) \in N_{1}$ and for all $(r, s) \in \mathbb{Z}^{2}$,

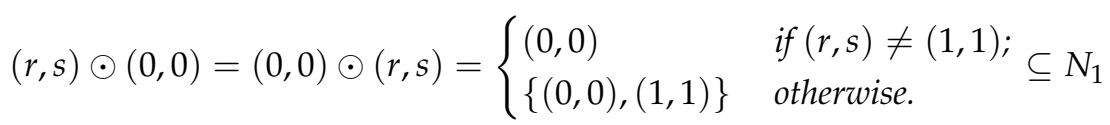

implies that $N_{1}$ is a NeutroHyperideal of $\mathbb{Z}^{2}$.

Remark 8. The intersection of NeutroHyperideals may fail to be a NeutroHyperideal. This is clear from Example 17 as $\{(0,0),(1,1)\}=N_{1} \cap N_{2}$ is not a NeutroHyperideal of $\mathbb{Z}^{2}$.

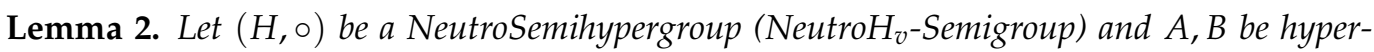
groupoids. If $A, B$ are NeutroLeftHyperideals (NeutroRightHyperideals or NeutroHyperideals) of $H$. Then $A \cup B$ is a NeutroLeftHyperideal (NeutroRightHyperideal or NeutroHyperideal) of $H$.

Proof. Let $A, B$ be NeutroLeftHyperideals of $H$. Lemma 1 asserts that $A \cup B$ is a NeutroSubsemihypergroup ( $\mathrm{NeutroH}_{v}-$ Subsemigroup) of $H$. Since $A$ is a NeutroLeftHyperideal 
of $H$, it follows that there exists $a \in A$ such that $r \circ a \subseteq A$ for all $r \in H$. The latter implies that there exists $a \in A \cup B$ such that $r \circ a \subseteq A \cup B$ for all $r \in H$. Thus, $A \cup B$ is a NeutroLeftHyperideal of $H$.

Definition 12. Let $(H, \circ),\left(H^{\prime}, \star\right)$ be NeutroSemihypergroups (NeutroH $H_{v}-S_{\text {Semigroups }}$ ) and $\phi$ : $H \rightarrow H^{\prime}$ be a function. Then

(1) $\phi$ is called NeutroHomomorphism if $\phi(x \circ y)=\phi(x) \star \phi(y)$ for some $x, y \in A$.

(2) $\phi$ is called NeutroIsomomorphism if $\phi$ is a bijective NeutroHomomorphism.

(3) $\phi$ is called NeutroStrongHomomorphism if for all $x, y \in A, \phi(x \circ y)=\phi(x) \star \phi(y)$ when $x \circ y \subseteq H, \phi(x) \star \phi(y) \nsubseteq H^{\prime}$ when $x \circ y \nsubseteq H$, and $\phi(x) \star \phi(y)$ is indeterminate when $x \circ y$ is indeterminate.

(4) $\phi$ is called NeutroStrongIsomomorphism if $\phi$ is a bijective NeutroOrderedStrongHomomorphism. In this case we say that $(H, \circ) \cong{ }_{S I}\left(H^{\prime}, \star\right)$.

Example 18. Let $(M, \bullet)$ and $(M, \circledast)$ be the NeutroSemihypergroups defined in Examples 11 and 13 , respectively. Then $(M, \bullet) \cong S I(M, \circledast)$ as $\phi:(M, \bullet) \rightarrow(M, \circledast)$ is a NeutroStongIsomorphism. Here,

$$
\phi(m)=a, \phi(a)=m, \text { and } \phi(d)=d .
$$

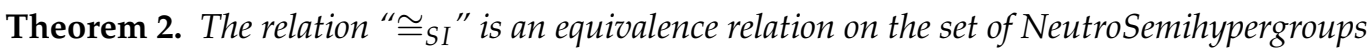

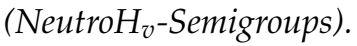

Proof. By taking the identity map, we can easily prove that " $\cong S I$ " is a reflexive relation. Let $A \cong{ }_{S I} B$. Then there exists a NeutroStrongIsomorphism $\phi:(A, \star) \rightarrow(B, \circledast)$. We prove that the inverse function $\phi^{-1}: B \rightarrow A$ of $\phi$ is a NeutroStrongIsomorphism. For all $b_{1}, b_{2} \in B$, there exist $a_{1}, a_{2} \in A$ with $\phi\left(a_{1}\right)=b_{1}$ and $\phi\left(a_{2}\right)=b_{2}$. We have

$$
\phi^{-1}\left(b_{1} \circledast b_{2}\right)=\phi^{-1}\left(\phi\left(a_{1}\right) \circledast \phi\left(a_{2}\right)\right)
$$

We consider the following cases for $\phi\left(a_{1}\right) \circledast \phi\left(a_{2}\right)$.

Case $\phi\left(a_{1}\right) \circledast \phi\left(a_{2}\right) \subseteq B$. Having $\phi$ a NeutroStrongIsomorphism and $\phi\left(a_{1}\right) \circledast \phi\left(a_{2}\right) \subseteq$ $B$ imply that $a_{1} \star a_{2} \subseteq A$ and hence,

$$
\phi^{-1}\left(b_{1} \circledast b_{2}\right)=\phi^{-1}\left(\phi\left(a_{1}\right) \circledast \phi\left(a_{2}\right)\right)=\phi^{-1}\left(\phi\left(a_{1} \star a_{2}\right)\right)=a_{1} \star a_{2}=\phi^{-1}\left(b_{1}\right) \star \phi^{-1}\left(b_{2}\right) .
$$

Case $\phi\left(a_{1}\right) \circledast \phi\left(a_{2}\right) \nsubseteq B$. Suppose, to get contradiction, that $\phi^{-1}\left(\phi\left(a_{1}\right)\right) \star \phi^{-1}\left(\phi\left(a_{2}\right)\right)=$ $a_{1} \star a_{2} \subseteq A$ or indeterminate. Then by using our hypothesis that $\phi$ is NeutroStrongIsomorphism, we get that $\phi\left(a_{1}\right) \circledast \phi\left(a_{2}\right) \subseteq B$ or indeterminate.

Case $\phi\left(a_{1}\right) \circledast \phi\left(a_{2}\right)$ is indeterminate. Suppose, to get contradiction, that $\phi^{-1}\left(\phi\left(a_{1}\right)\right) \star$ $\phi^{-1}\left(\phi\left(a_{2}\right)\right)=a_{1} \star a_{2} \subseteq A$ or $a_{1} \star a_{2} \nsubseteq A$. Then by using our hypothesis that $\phi$ is NeutroStrongIsomorphism, we get that $\phi\left(a_{1}\right) \circledast \phi\left(a_{2}\right) \subseteq B$ or $\phi\left(a_{1}\right) \circledast \phi\left(a_{2}\right) \nsubseteq B$.

Thus, $B \cong_{S I} A$ and hence, " $\cong_{S I}$ " is a symmetric relation. Let $A \cong_{S I} B$ and $B \cong_{S I} C$. Then there exist NeutroStrongIsomorphisms $\phi: A \rightarrow B$ and $\psi: B \rightarrow C$. One can easily see that the composition function $\psi \circ \phi: A \rightarrow C$ of $\psi$ and $\phi$ is a NeutroStrongIsomorphism. Thus, $A \cong_{S I} C$ and hence, " $\cong S I "$ is a transitive relation.

Lemma 3. Let $(H, \circ),\left(H^{\prime}, \star\right)$ be NeutroSemihypergroups (NeutroH ${ }_{v}$ Semigroups) and $\phi$ : $H \rightarrow H^{\prime}$ be an injective NeutroStrongHomomorphism. If $M \subset H$ is a NeutroSubsemihyper-

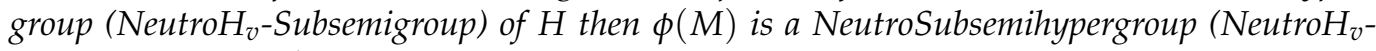
Subsemigroup) of $H^{\prime}$.

Proof. Let $M$ be a NeutroSubsemihypergroup of $H$. If "o" is NeutroHyperoperation on $M$ then it is clear that " $\star$ " is NeutroHyperoperation on $\phi(M)$. If "०" is NeutroAssociative 
then there exist $x, y, z, a, b, c, d, e, f \in$ Msatisfying some (or all) of the following conditions in a way that $(T, I, F) \notin\{(1,0,0),(0,0,1)\}$.

1. $T: x \circ(y \circ z)=(x \circ y) \circ z$;

2. $F: a \circ(b \circ c) \neq(a \circ b) \circ c$;

3. I: $e \circ(f \circ g)$ is indeterminate or $(e \circ f) \circ g$ is indeterminate or we cannot find if $e \circ(f \circ g)$ and $(e \circ f) \circ g$ are equal.

The latter and having $\phi$ an injective NeutroStrongHomomorphism imply that some (or all) of the following conditions are satisfied in a way that $(T, I, F) \notin\{(1,0,0),(0,0,1)\}$.

1. $T: \phi(x) \star(\phi(y) \star \phi(z))=(\phi(x) \star \phi(y)) \star \phi(z) ;$

2. $F: \phi(a) \star(\phi(b) \star \phi(c)) \neq(\phi(a) \star \phi(b)) \star \phi(c)$;

3. I: $\phi(e) \star(\phi(f) \star \phi(g))$ is indeterminate or $(\phi(e) \star \phi(f)) \star \phi(g)$ is indeterminate or we cannot find if $\phi(e) \star(\phi(f) \star \phi(g))$ and $(\phi(e) \star \phi(f)) \star \phi(g)$ are equal.

Thus, $\phi(M)$ is a NeutroSubsemihypergroup. The proof that $\phi(M)$ is a $\mathrm{NeutroH}_{v^{-}}$ Subsemigroup of $H^{\prime}$ is done similarly.

Example 19. Let $(M, \bullet)$ and $(M, \circledast)$ be the NeutroSemihypergroups defined in Examples 11 and 13, respectively. Example 15 asserts that $\{m, a\}$ is a NeutroSubsemihypergroup of $(M, \bullet)$. Using Example 18 and Lemma 3, we get that $\{a, m\}=\{\phi(m), \phi(a)\}$ is a NeutroSubsemihypergroup of $(M, \circledast)$.

Lemma 4. Let $(H, \circ),\left(H^{\prime}, \star\right)$ be NeutroSemihypergroups (NeutroH ${ }_{v}$-Semigroups) and $\phi: H \rightarrow$ $H^{\prime}$ be a NeutroStrongIsomomorphism. If $N \subseteq H^{\prime}$ is a NeutroSubsemihypergroup (Neutro $\mathrm{H}_{v^{-}}$ Subsemigroup) of $H^{\prime}$ then $\phi^{-1}(N)$ is a NeutroSubsemihypergroup (Neutro $H_{v}$-Subsemigroup) of $H$.

Proof. Let $N \subset H^{\prime}$ be a NeutroSubsemihypergroup of $H^{\prime}$. If " $\star$ " is NeutroHyperoperation on $N$ then it is clear that "o" is NeutroHyperoperation on $\phi^{-1}(N)$. Let " $\star$ " be NeutroAssociative. Having $\phi$ is an onto NeutroStrongHomomorphism implies that there exist $\phi(x), \phi(y), \phi(z), \phi(a), \phi(b), \phi(c), \phi(d), \phi(e), \phi(f) \in N$ satisfying some (or all) of the following conditions in a way that $(T, I, F) \notin\{(1,0,0),(0,0,1)\}$.

1. $T: \phi(x) \star(\phi(y) \star \phi(z))=(\phi(x) \star \phi(y)) \star \phi(z)$;

2. $F: \phi(a) \star(\phi(b) \star \phi(c)) \neq(\phi(a) \star \phi(b)) \star \phi(c)$;

3. I: $\phi(e) \star(\phi(f) \star \phi(g))$ is indeterminate or $(\phi(e) \star \phi(f)) \star \phi(g)$ is indeterminate or we cannot find if $\phi(e) \star(\phi(f) \star \phi(g))$ and $(\phi(e) \star \phi(f)) \star \phi(g)$ are equal.

Having $\phi$ be an injective NeutroStrongHomomorphism implies that there exist $x, y, z, a$, $b, c, d, e, f \in \phi^{-1}(N)$ satisfying some (or all) of the following conditions in a way that $(T, I, F) \notin\{(1,0,0),(0,0,1)\}$.

1. $T: x \circ(y \circ z)=(x \circ y) \circ z$;

2. $F: a \circ(b \circ c) \neq(a \circ b) \circ c$;

3. I: $e \circ(f \circ g)$ is indeterminate or $(e \circ f) \circ g$ is indeterminate or we cannot find if $e \circ(f \circ g)$ and $(e \circ f) \circ g$ are equal.

Thus, $\phi^{-1}(N)$ is a NeutroSubsemihypergroup of $H$. The proof that $\phi^{-1}(N)$ is a $\mathrm{NeutroH}_{v}$-Subsemigroup of $H$ may be done similarly.

Theorem 3. Let $(H, \circ),\left(H^{\prime}, \star\right)$ be NeutroSemihypergroups (Neutro $H_{v}$-Semigroups) and $\phi: H \rightarrow$ $H^{\prime}$ be a NeutroStrongIsomorphism. Then $M \subseteq H$ is a NeutroSubsemihypergroup (NeutroH $H_{v^{-}}$ Subsemigroup) of $\mathrm{H}$ if and only if $\phi(M)$ is a NeutroSubsemihypergroup (Neutro $\mathrm{H}_{v}$-Subsemigroup) of $\mathrm{H}^{\prime}$.

Proof. The proof follows from Theorem 2 and Lemmas 3 and 4.

Corollary 1. Let $(H, \circ),\left(H^{\prime}, \star\right)$ be NeutroSemihypergroups (NeutroH ${ }_{v}$-Semigroups) and $\phi$ : $H \rightarrow H^{\prime}$ be a NeutroStrongIsomorphism. Then $H$ is simple if and only if $H^{\prime}$ is simple. 
Proof. The proof follows from Theorem 3.

Lemma 5. Let $(H, \circ),\left(H^{\prime}, \star\right)$ be NeutroSemihypergroups (NeutroH $H_{v}$-Semigroups) and $\phi: H \rightarrow$ $H^{\prime}$ be a NeutroStrongIsomorphism. If $M \subseteq H$ is a NeutroLeftHyperideal (NeutroRightHyperideal or NeutroHyperideal) of $H$ then $\phi(M)$ is a NeutroLeftHyperideal (NeutroRightHyperideal or NeutroHyperideal) of $\mathrm{H}^{\prime}$.

Proof. Let $M \subseteq H$ be a NeutroLeftHyperideal of $H$. Lemma 3 asserts that $\phi(M)$ is a NeutroSubsemihypergroup (NeutroH ${ }_{v}$-Subsemigroup) of $H^{\prime}$. Having $M$ a NeutroLeftHyperideal of $H$ implies that there exists $x \in M$ such that $r \circ x \subseteq M$ for all $r \in H$. Having $\phi$ an onto NeutroStrongHomomorphism implies that $\phi(r) \star \phi(x) \subseteq \phi(M)$ for all $s=\phi(r) \in H^{\prime}$. Thus, $\phi(M)$ is a NeutroLeftHyperideal of $H^{\prime}$. The proofs of NeutroRightHyperideal and NeutroHyperideal are done similarly.

Lemma 6. Let $(H, \circ),\left(H^{\prime}, \star\right)$ be NeutroSemihypergroups (NeutroH ${ }_{v}$-Semigroups) and $\phi: H \rightarrow$ $H^{\prime}$ be a NeutroStrongIsomorphism. If $N \subseteq H^{\prime}$ is a NeutroLeftHyperideal (NeutroRightHyperideal or NeutroHyperideal) of $H^{\prime}$ then $\phi^{-1}(N)$ is a NeutroLeftHyperideal (NeutroRightHyperideal or NeutroHyperideal) of $\mathrm{H}$.

Proof. Let $N \subseteq H^{\prime}$ be a NeutroLeftHyperideal of $H$. Lemma 3 asserts that $\phi^{-1}(N)$ is a NeutroSubsemihypergroup ( $\mathrm{NeutroH}_{v}$-Subsemigroup) of $H$. Having $N$ a NeutroLeftHyperideal of $H^{\prime}$ implies that there exists $y \in N$ such that $s \star y \subseteq N$ for all $s \in H^{\prime}$. Since $\phi$ is an NeutroStrongHomomorphism, it follows that $\phi(r \circ x) \subseteq N$ for all $r \in H$ where $y=\phi(x)$. The latter implies that there exists $x \in \phi^{-1}(N)$ with $r \circ x \subseteq \phi^{-1}(N)$ for all $r \in H$. Thus, $\phi^{-1}(N)$ is a NeutroLeftHyperideal of $H$. The proofs of NeutroRightHyperideal and NeutroHyperideal are done similarly.

Theorem 4. Let $(H, \circ),\left(H^{\prime}, \star\right)$ be NeutroSemihypergroups (NeutroH $H_{v}$-Semigroups) and $\phi$ : $H \rightarrow H^{\prime}$ be a NeutroStrongIsomorphism. Then $M \subseteq H$ is a NeutroLeftHyperideal (NeutroRightHyperideal or NeutroHyperideal) of $H$ if and only if $\phi(M)$ is a NeutroLeftHyperideal (NeutroRightHyperideal or NeutroHyperideal) of $H^{\prime}$.

Proof. The proof follows from Theorem 2, Lemmas 5 and 6.

Let $H_{\alpha}$ be any non-empty set for all $\alpha \in \Gamma$ and ". $\alpha$ " be a hyperoperation on $H_{\alpha}$. We define "o" on $\prod_{\alpha \in \Gamma} H_{\alpha}$ as follows: For all $\left(x_{\alpha}\right),\left(y_{\alpha}\right) \in \prod_{\alpha \in \Gamma} H_{\alpha},\left(x_{\alpha}\right) \circ\left(y_{\alpha}\right)=\left\{\left(t_{\alpha}\right): t_{\alpha} \in\right.$ $\left.x_{\alpha} \cdot \alpha y_{\alpha}\right\}$.

Theorem 5. Let $\left(H_{1}, \circ_{1}\right)$ and $\left(H_{2}, o_{2}\right)$ be hypergroupoids. Then $\left(H_{1} \times H_{2}, \circ\right)$ is a NeutroSemihypergroup (NeutroH $\mathrm{H}_{v}$-Semigroup) if and only if either $\left(\mathrm{H}_{1}, \mathrm{o}_{1}\right)$ is a NeutroSemihypergroup ( $\mathrm{NeutroH}_{v}$-Semigroup) or $\left(\mathrm{H}_{2}, \mathrm{O}_{2}\right)$ is a NeutroSemihypergroup (NeutroH $\mathrm{H}_{v}$-Semigroup) or both are NeutroSemihypergroups (NeutroH $\mathrm{H}_{v}$-Semigroups).

Proof. The proof is straightforward.

Example 20. Let $(\mathbb{R}, *)$ be the semihypergroup defined as: $x * y=\{x, y\}$ for all $x, y \in \mathbb{R}$ and $(M, \cdot)$ be the NeutroSemihypergroup defined in Example 6. Then the following are true.

1. $(\mathbb{R} \times M, 0)$ is a NeutroSemihypergroup,

2. $(M \times \mathbb{R}, 0)$ is a NeutroSemihypergroup, and

3. $(M \times M, \circ)$ is a NeutroSemihypergroup.

In what follows, we present a way to construct a new NeutroSemihypergroup ( $\mathrm{NeutroH}_{V^{-}}$ Semigroup) from an existing one. This tool is of great importance to prove that for any positive integer $n \geq 2$, there exists at least one NeutroSemihypergroup ( $\mathrm{NeutroH}_{v}$-Semigroup) of order $n$. 
Let $(H, \circ)$ be a NeutroSemihypergroup $\left(\mathrm{NeutroH}_{v}\right.$-Semigroup) and $J$ be any nonempty set such that $H \cap J=\varnothing$ and $(H \circ H) \cap J=\varnothing$. The extension $H[J]$ of $H$ by $J$ is given as $H[J]=H \cup J$. We define the hyperoperation "๑" on $H[J]$ as follows.

$$
x \odot y= \begin{cases}x \circ y & \text { if } x, y \in H ; \\ H \cup J & \text { otherwise. }\end{cases}
$$

Theorem 6. Let $(H, \circ)$ be a NeutroSemihypergroup (NeutroH ${ }_{v}$-Semigroup) and J be any nonempty set such that $H \cap J=\varnothing$ and $(H \circ H) \cap J=\varnothing$. Then $(H[J], \odot)$ is a NeutroSemihypergroup (NeutroH ${ }_{v}$-Semigroup).

Proof. Let $(H, \circ)$ be a NeutroSemihypergroup. If "o" is a NeutroHyperoperation then there exist $u, v, w, x, y, z \in H$ with $u \circ v \subseteq H$ representing " $\mathrm{T}^{\prime}, w \circ x \nsubseteq \not H$ representing " $\mathrm{F}$ ", $y \circ z$ is indeterminate representing " $\mathrm{I}$ ". Where $(T, I, F) \notin\{(1,0,0),(0,0,1)\}$. Since $(H \circ H) \cap J=\varnothing$, it follows that there exist $u, v, w, x, y, z \in H$ with $u \circ v \subseteq H[J]$ representing " $\mathrm{T}$ ", $w \circ x \nsubseteq H[J]$ representing " $\mathrm{F}$ " (as $w \circ x \nsubseteq H$ and $w \circ x \nsubseteq J), y \circ z$ is indeterminate representing "I". Where $(T, I, F) \notin\{(1,0,0),(0,0,1)\}$. Thus, “๑” is NeutroHyperoperation on $H[J]$. If " $\circ$ " is NeutroAssociative on $H$ then it is clear that "๑)" is NeutroAssociative on $H[J]$. Therefore, $(H[J], \odot)$ is a NeutroSemihypergroup. The case $(H[J], \odot)$ is a $\mathrm{NeutroH}_{v^{-}}$ Semigroup is done similarly.

Example 21. Let $(M, \cdot)$ be the NeutroSemihypergroup defined in Example 6 and $N=\{n\}$. Then $M[N]=\{m, a, d, n\}$ and $(M[N], \odot)$ is the NeutroSemihypergroup defined by the following table.

\begin{tabular}{|c|cccc|}
\hline$\odot$ & $m$ & $a$ & $d$ & $n$ \\
\hline$m$ & $m$ & $m$ & $m$ & $\{m, a, d, n\}$ \\
$a$ & $m$ & $\{m, a\}$ & $d$ & $\{m, a, d, n\}$ \\
$d$ & $m$ & $d$ & $d$ & $\{m, a, d, n\}$ \\
$n$ & $\{m, a, d, n\}$ & $\{m, a, d, n\}$ & $\{m, a, d, n\}$ & $\{m, a, d, n\}$ \\
\hline
\end{tabular}

Theorem 7. Let $n \geq 2$ be an integer. Then there is at least one NeutroSemihypergroup of order $n$.

Proof. The proof follows from Example 4 and Theorem 6 .

Corollary 2. There are infinitely many NeutroSemihypergroups up to NeutroStrongIsomorphism.

Proof. The proof follows from Theorem 7.

Theorem 8. Let $n \geq 2$ be any integer. Then there is at least one $\mathrm{NeutroH}_{v}$-Semigroup of order $n$.

Proof. The proof follows from Example 4 and Theorem 6.

Corollary 3. There are infinitely many $\mathrm{NeutroH}_{v}$-Semigroups up to NeutroStrongIsomorphism.

Proof. The proof follows from Theorem 8.

\section{Conclusions}

In this paper, we discussed the properties of some NeutroHyperstructures. More precisely, we introduced NeutroSemihypergroups ( $\mathrm{NeutroH}_{v}$-Semigroups), constructed several examples, and studied some of their important subsets under NeutroStrongIsomorphism. It was shown through examples that some of the well known results for algebraic hyperstructures do not hold for NeutroHyperstructures. Moreover, it was proved that there is at least one NeutroSemihypergroup ( $\mathrm{NeutroH}_{v}$-Semigroups) of order $n$ where $n$ is any integer greater than one. The results in this paper may be considered as a base for any possible study in the field of NeutroHyperstructures. 
For future research, we raise the following ideas.

1. Find all NeutroSemihypergroups ( $\mathrm{NeutroH}_{v}$-Semigroups) of small order (up to NeutroStrongIsomorphism).

2. Find bounds for the number of finite NeutroSemihypergroups ( $\mathrm{NeutroH}_{v}$-Semigroups) of arbitrary order $n$ (up to NeutroStrongIsomorphism).

3. Classify simple NeutroSemihypergroups ( $\mathrm{NeutroH}_{v}$-Semigroups) up to NeutroStrongIsomorphism.

4. Define other NeutroHyperstructures such as NeutroPolygroup, NeutroHyperring, etc.

5. Find applications of NeutroHyperstructures in some fields like Biology, Physics, Chemistry, etc.

Author Contributions: Conceptualization, M.A.-T., B.D., F.S., and O.A.; methodology, M.A.-T., B.D., F.S., and O.A.; writing—original draft preparation, M.A.-T.; writing—review and editing, M.A.-T., B.D., F.S., and O.A. All authors have read and agreed to the published version of the manuscript.

Funding: This research received no external funding.

Institutional Review Board Statement: Not applicable.

Informed Consent Statement: Not applicable.

Data Availability Statement: Not applicable.

Conflicts of Interest: The authors declare no conflict of interest.

\section{References}

1. Smarandache, F. NeutroAlgebra is a Generalization of Partial Algebra. Int. J. Neutrosophic Sci. IJNS 2020, 2, 8-17. Available online: http:/ / fs.unm.edu/NeutroAlgebra.pdf (accessed on 1 January 2020).

2. Smarandache, F. Introduction to NeutroAlgebraic Structures and AntiAlgebraic Structures. In Advances of Standard and Nonstandard Neutrosophic Theories; Pons Publishing House: Brussels, Belgium, 2019; Chapter 6, pp. 240-265. Available online: http:/ /fs.unm. edu/AdvancesOfStandardAndNonstandard.pdf (accessed on 1 January 2020).

3. Smarandache, F. Introduction to NeutroAlgebraic Structures and AntiAlgebraic Structures (revisited). Neutrosophic Sets Syst. 2020, 31, 1-16. [CrossRef]

4. Agboola, A.A.A. Introduction to NeutroGroups. Int. J. Neutrosophic Sci. IJNS 2020, 6, 41-47.

5. Agboola, A.A.A. Introduction to NeutroRings. Int. J. Neutrosophic Sci. IJNS 2020, 7, 62-73.

6. Al-Tahan, M.; Smarandache, F.; Davvaz, B. NeutroOrderedAlgebra: Applications to Semigroups. Neutrosophic Sets Syst. $2021,39,133-147$.

7. Al-Tahan, M.; Davvaz, B.; Smarandache, F.; Osman, O. On Some Properties of Productional NeutroOrderedSemigroups. 2021, submitted.

8. Hamidi, M.; Smarandache, F. Neutro-BCK-Algebra. Int. J. Neutrosophic Sci. IJNS 2020, 8, 110-117.

9. Rezaei, A.; Smarandache, F. On Neutro-BE-algebras and Anti-BE-algebras. Int. J. Neutrosophic Sci. IJNS 2020, 4, 8-15.

10. Smarandache, F.; Rezaei, A.; Kim, H.S. A New Trend to Extensions of CI-algebras. Int. J. Neutrosophic Sci. IJNS 2020, 5, 8-15. [CrossRef]

11. Marty, F. Sur une generalization de la notion de groupe. In Proceedings of the 8th Congress on Mathmatics, Scandinaves, Stockholm, Sweden, 14-18 August 1934; pp. 45-49.

12. Al-Tahan, M.; Davvaz, B. Chemical Hyperstructures for Astatine, Tellurium and for Bismuth. Bull. Comput. Appl. Math. $2019,7,9-25$.

13. Al-Tahan, M.; Davvaz, B. On the Existence of Hyperrings Associated to Arithmetic Functions. J. Number Theory 2017, 174, 136-149. [CrossRef]

14. Al-Tahan, M.; Davvaz, B. Algebraic Hyperstructures Associated to Biological Inheritance. Math. Biosci. 2017, 285, 112-118. [CrossRef]

15. Davvaz, B.; Subiono; Al-Tahan, M. Calculus of Meet Plus Hyperalgebra (Tropical Semihyperrings). Commun. Algebra 2020, 48. [CrossRef]

16. Corsini, P. Prolegomena of Hypergroup Theory; Udine Aviani Editore: Tricesimo (Udine), Italy, 1993.

17. Davvaz, B. Polygroup Theory and Related Systems; World Scientific Publishing Co., Pte. Ltd.: Hackensack, NJ, USA, 2013; viii+200p.

18. Davvaz, B.; Leoreanu-Fotea, V. Hyperring Theory and Applications; International Academic Press: Cambridge, MA, USA, 2008.

19. Vougiouklis, T. The Fundamental Relation in Hyperrings. The General Hyperfield. In Proceedings of the Fourth International Congress on Algebraic Hyperstructures and Applications (AHA 1990); World Scientific: Singapore, 1991; pp. $203-211$.

20. Vougiouklis, T. Hyperstructures and Their Representations; Hadronic Press, Inc.: Palm Harber, FL, USA, 1994.

21. Vougiouklis, T. $H_{v}$-groups Defined on the Same Set. Discret. Math. 1996, 155, 259-265. [CrossRef]

22. Vougiouklis, T.; Spartalis, S.; Kessoglides, M. Weak Hyperstructures on Small Sets. Ratio Math. 1997, 12, 90-96.

23. Ibrahim, M.A.; Agboola, A.A.A. Introduction to NeutroHyperGroups. Neutrosophic Sets Syst. 2020, 38, 15-32.

24. Smarandache, F. Extension of HyperGraph to n-SuperHyperGraph and to Plithogenic n-SuperHyperGraph, and Extension of HyperAlgebra to n-ary (Classical-/Neutro-/Anti-)HyperAlgebra. Neutrosophic Sets Syst. 2020, 33, $290-296$. 